\title{
Reproducibility of sensory testing and voluntary muscle testing in evaluating the treatment of acute neuritis in leprosy patients *
}

\author{
SUSAN LEWIS \\ Department of Medicine, University Hospital, Queen's Medical \\ Centre, Nottingham NG7 $2 U H$
}

Received for publication 7 August 1981

\begin{abstract}
Summary Based on methods discussed at a 'neuritis workshop' held in Karigiri in South India in 1980, this study describes attempts to standardize sensory testing and voluntary muscle testing in a group of 20 patients with tuberculoid leprosy. Patients were assessed by the same observer on two occasions at intervals of 2 weeks. The results confirm that accurate methods for assessing the treatment of neuritis in leprosy remain elusive. In this group of patients however, and under the conditions described, it appears that greater reliance can be placed on sensory testing, particularly fine touch, than on voluntary muscle testing.
\end{abstract}

\section{Introduction}

One of the most controversial problems in leprosy research is the treatment of acute neuritis and how best to evaluate its effectiveness. Sensory testing has been advocated: ${ }^{1}$ von Frey hairs of graded sizes were used, exerting pressure of $0.1 \mathrm{~g}, 0.4 \mathrm{~g}, 1.0 \mathrm{~g}, 2 \mathrm{~g}$ and $4 \mathrm{~g}$. The thenar and hypothenar areas of the hand were touched four times with each stimulator. They recorded the numbers 'felts' and graded the numbers 'not felt'. They found this to be a sensitive method in the follow-up of nerve involvement.

Voluntary muscle testing was found to be a reliable and reproducible method. ${ }^{2}$ The Medical Research Council (MRC) scale of strength was used to give a score for seven muscles supplied by the ulnar nerve and nine muscles supplied by the median nerve. In conjunction with nerve conduction velocity (NCV)studies, both sensory testing and voluntary muscle testing have been used to evaluate the success of treatment. ${ }^{3,4}$ At the Neuritis Workshop in Karigiri, South India ${ }^{5}$ an attempt was made to standardize these tests. The object of this

*Study done at the Schieffelin Leprosy Research and Training Centre, Karigiri, South India, during a student elective period May 1980.

$0305-7518 / 83 / 054023+08 \$ 1.00$ @ British Leprosy Relief Association 
study is to examine the reproducibility of sensory and voluntary muscle testing, using some of the methods recommended at the Workshop.

\section{Methods}

Twenty patients with tuberculoid leprosy, as clinically diagnosed, with established nerve lesions were assessed by the author on two occasions, separated by 1 to 3 weeks. Both hands were examined for ulnar and median nerve function using sensory and voluntary muscle testing. Ulnar nerve damage was considerably more common than median nerve damage.

\section{Sensory testing (ST)}

Four methods were used: light touch, static two-point discrimination, tuning fork test and pinprick test. Six areas were examined on the palmar surface of each hand (Fig. 1). Ulnar nerve supplied: 1, distal phalanx - little finger; 2, proximal phalanx - little finger; 3, hypothenar eminance. Median nerve supplied: 1 , distal phalanx - index finger; 2 , proximal phalan $\mathrm{x}$ - index finger; 3, distal pulp - thumb.

\section{Light touch}

Four stimulators were constructed from nylon hairs which bent at a pressure of $0.5 \mathrm{~g}, 3.5 \mathrm{~g}, 5.5 \mathrm{~g}, 30 \mathrm{~g}$.

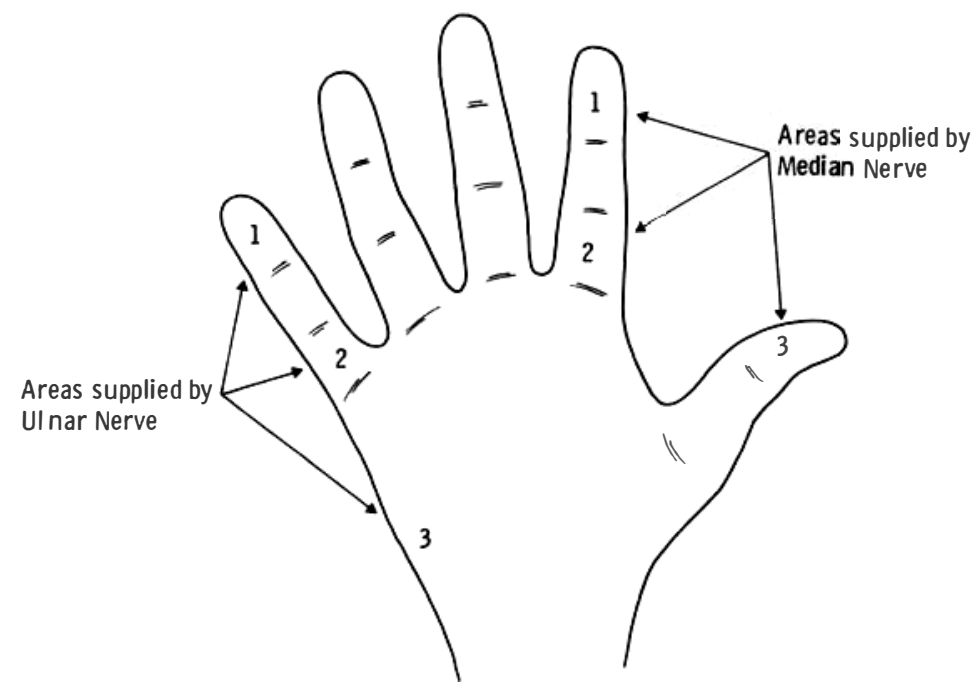

Figure 1. 
The finest hair that was felt in each area was recorded and scored as: $0.5 \mathrm{~g}=4 ; 3.5 \mathrm{~g}=3 ; 5.5 \mathrm{~g}=2 ; 30 \mathrm{~g}=1$; not felt $=0$. Thus the maximum possible score for one nerve was 12 points.

\section{Static two-point discrimination}

Three paper-clips were used, with the legs bent at $3 \mathrm{~mm}, 6 \mathrm{~mm}$ and $12 \mathrm{~mm}$ apart. The six areas were used as before and the shortest distance felt as two separate stimuli was noted and scored for each site as: $3 \mathrm{~mm}=3 ; 6 \mathrm{~mm}=2$; $12 \mathrm{~mm}=1$; not felt or felt as one $=0$. Thus the maximum score for a single nerve was 9 points.

\section{Tuning fork test}

The vibrating arm of a $256 \mathrm{~Hz}$ tuning fork was touched lightly in each area and a point awarded if the patient could feel the vibration. Thus the maximum score for a single nerve was 3 points.

\section{Pinprick test}

The patient was asked to state whether the sensation was sharp or blunt. The result was recorded for each test site as: $\operatorname{sharp}=3$; blunt $=0$; not felt $=0$. Thus the maximum score for each nerve was 9 points. The sum of the scores for each test gave a score out of 33 for the function of each nerve.

It must be emphasized that even when a nerve is unaffected by leprosy, the score may not reach 33. Tests of two-point discrimination showed performance varied with age and that volar fingertips were twice as discriminating as the thenar and hypothenar areas. ${ }^{6}$ Farm labourers with thick skin were often unable to feel the finest hair on the palms of their hands when there was no subjective loss of sensation. Thus a 'normal score' varied between 28 and 33 . It is not the absolute score that was of interest in this study but the reproducibility of the scoring methods.

\section{Voluntary muscle testing (VMT)}

VMT consisted of estimation of muscle power in eight muscles in each hand, four supplied by the ulnar nerve and four supplied by the median nerve. The power was graded according to the MRC scale of strength:

5 Full strength against resistance provided by the assessor's hand or finger.

4 Movement against some resistance is possible. Range of movement is full.

3 There is a full range of movement, not possible against resistance (but can be done against gravity).

2 There is less than full range of movement. 
1 A flicker of movement or muscle contraction can be felt but no actual joint movement.

0 No movement.

Muscles examined were flexor carpi ulnaris, abductor digiti minimi, first
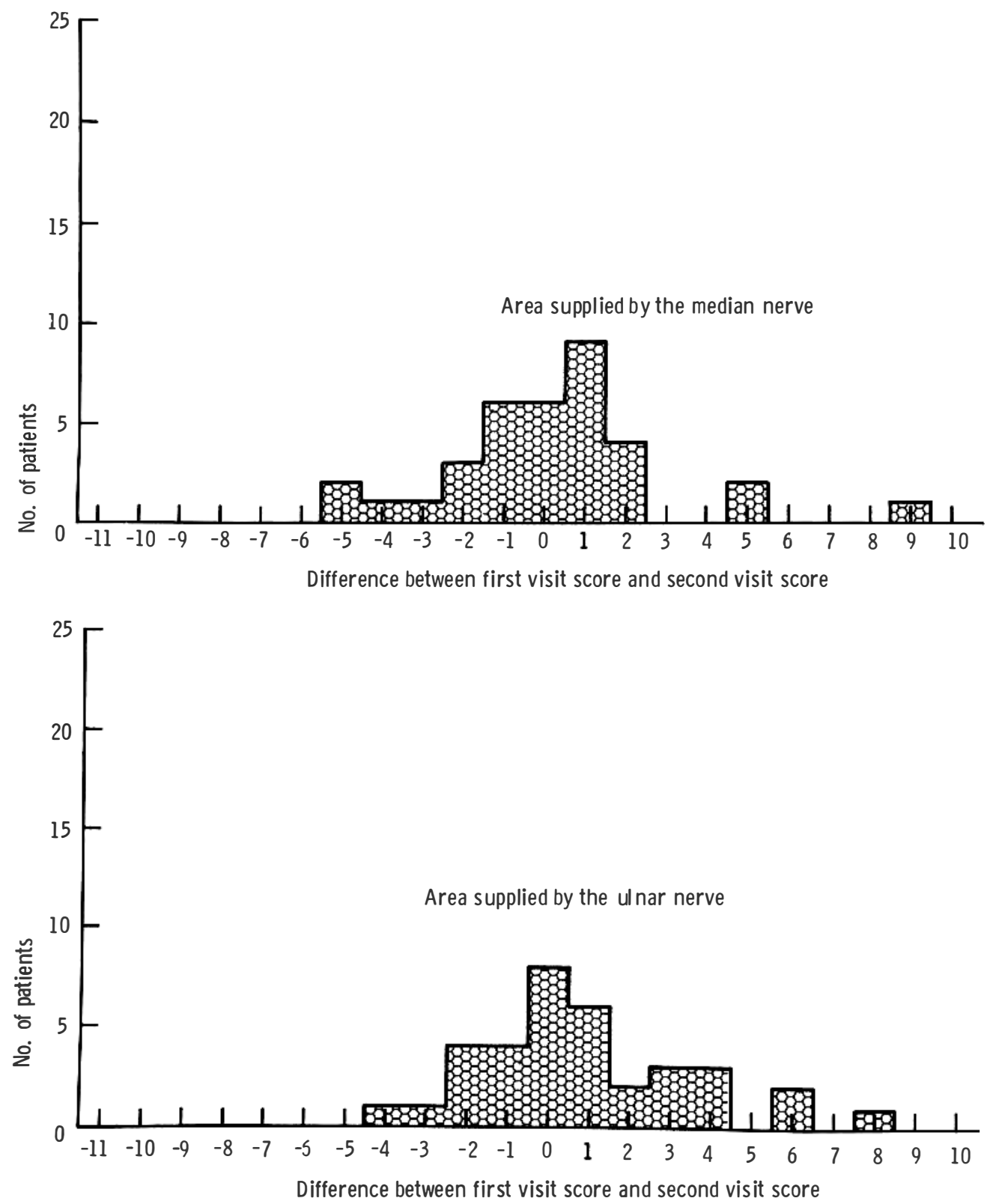

Figure 2. Graph to show the reproducibility of the sensory testing method. 
dorsal interosseus, third palmar interosseus (supplied by the ulnar nerve) and flexor carpi radialis, flexor pollicis longus, abductor pollicis brevis and the first lumbrical (supplied by the median nerve). Thus as each muscle was given a score of up to five points and four muscles were assessed for each nerve the maximum score for an intact ulnar or median nerve was 20 points. This test
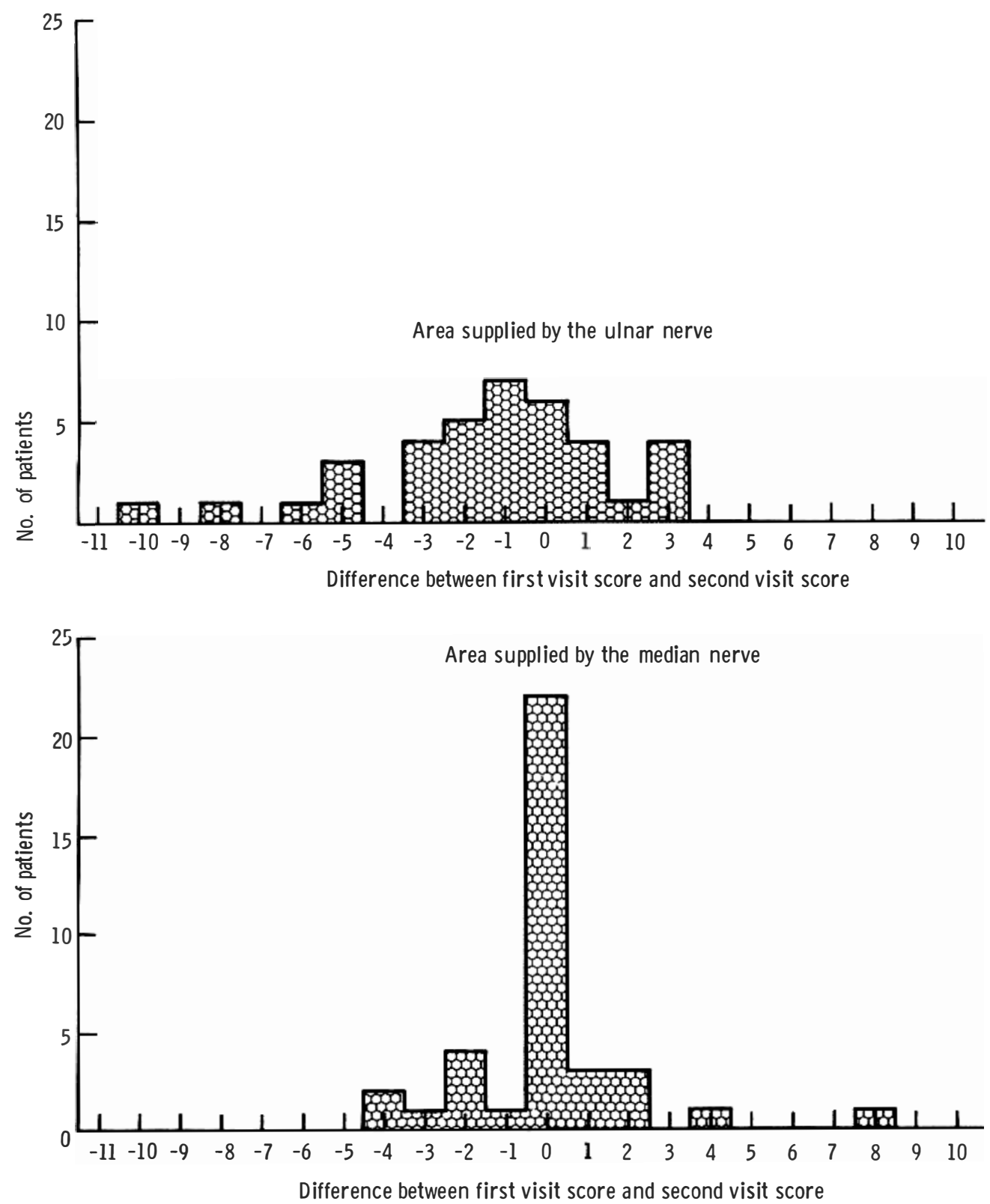

Figure 3. Graph showing the reproducibility of the voluntary muscle testing method. 
required more positive cooperation from the patients and a greater opportunity for observer error. It is difficult to assign five grades of strength when dealing with small muscles of the hand and requires practice to learn the normal strength in the hand of, for example, an old woman and a young man.

\section{Results}

The results consist of a score for ulnar and median nerve function as assessed on VMT and ST. The score achieved on the first visit was compared with that achieved on the second visit using Wilcoxon matched pairs signed ranks test for nonparametric data. ${ }^{7}$

There was found to be no significant difference between the sensory testing scores achieved on the first and second visit in both ulnar and median nerve groups, implying that the method used is reproducible and thus useful. Fine touch score seemed to be most sensitive of the four sensory testing methods used, the scores (out of 12) were compared and also found not to differ significantly. When muscle power was assessed the median nerve group showed no difference in score but the ulnar nerve group showed a significant difference (probability $<5 \% Z=2.019$ ). As the patients had established nerve lesions, tested twice at an interval of only 1 to 3 weeks, this result suggests that this method of assessment is not reliably reproducible. The differences between the first and second scores are illustrated graphically (Figs 2 and 3).

These results confirm the subjective opinion of the author that sensory testing, using a combination of tests described above, is a useful and reproducible method of assessing nerve function in leprosy patients. However, the test does have disadvantages - it is time consuming (each patient requiring up to 20 minutes), requires the full concentration of both patient and assessor and preferably the same assessor on each occasion. However, it is a method valuable in the study of the treatment of neuritis. A strength grade was difficult to assess in the small muscles of the hand and sensory testing was more accurate and useful.

\section{Discussion}

In this study, of patients with nerve damage due to leprosy, it was clear that pinprick sensation was present when there was loss of fine touch and that twopoint discrimination was lost early. Vibration (as tested with a tuning fork at $256 \mathrm{cps}$ ) was often present in the absence of pain sensation. It has been reported $^{8}$ when looking at recovery of sensation in the hand following nerve injury due to trauma that pain sensation was the first sensory modality to 
recover. However, it was also found that vibration at $30 \mathrm{cps}$ recovered before vibration at $256 \mathrm{cps}$, that moving touch recovered before constant touch awareness (slowly adapting fibres) and that last to recover was two-point discrimination.

One could speculate that the difference between these findings might be due to an idiosyncratic preservation of one sensory modality in leprosy neuritis or, more probably, the vibrating tuning fork was too diffuse a test, which recruited nerve endings from a larger area of the hand than that being tested. There may be a qualitative difference between sensory loss due to leprosy neuritis and that due to trauma: the 'dying back' phenomenon has been described. ${ }^{9}$ It seems that neural and Schwann cell activity is impaired throughout the whole length of a nerve affected by leprosy at any point along its course. Nerve regeneration and neuroma formation does not occur. The qualitative nature of sensory loss in leprosy neuritis warrants further investigation. From the results of this study it seems that VMT is an unreliable method of monitoring nerve damage except in the presence of very gross changes in function. VMT is a difficult test, especially in patients with acute neuritis as they are in pain and reluctant to cooperate.

An accurate method of assessing the treatment of neuritis remains elusive. The most useful parameter seems to be a combination of VMT, NCV, ST and symptoms. However, this type of assessment is cumbersome and hard to standardize between different centres.

I would recommend greater reliance on sensory testing, particularly fine touch and motor conduction studies, rather than voluntary muscle testing.

\section{Acknowledgements}

I would like to thank Dr Frank Johnson, Professor J R A Mitchell, Mrs Shirley Tacey, Mr Steve Kolumban and the staff of the Physiotherapy Department at Karigiri, and LEPRA who financed my visit.

\section{References}

1 Naafs B, Dagne T. Sensory testing - a sensitive method in the follow up of nerve involvement. Int J Lepr, 1977; 45; 4: 364.

2 Goodwin GS. The use of voluntary muscle test in leprosy neuritis. Lepr Rev, 1968; 39; 4: 209.

3 Naafs B, Pearson JMH, Baar AJM. A follow up study of nerve lesions in leprosy during and after reaction using motor nerve conduction velocity. Int $J$ Lepr, 1976; 44; 1-2: 189. 


\section{$30 \quad$ Susan Lewis}

4 Naafs B, Pearson JMH, Wheate HW. Reversal reaction - the prevention of permanent nerve damage. Comparison of short and long term steroid treatment. Int J Lepr, 1979; $47 ; 1$.

5 Neuritis Workshop Internal Publication of Schiefflin Leprosy Research and Training Centre, Karigiri, S. India. March 1980.

6 Gellis M, Pool R. Two point discrimination distances in the normal hand and forearm. Plastic \& reconstr surg, 1977; 59; 1: 57.

7 Bliss CI. Statistics in biology. McGraw Hill 1967: 226.

8 Dellon AL, Curtis R M, Edgerton MT. Evaluating recovery of sensation in the hand following nerve injury. Hopkins Med, 1972; 130: 235.

9 Weddell G. Disorders of peripheral cutaneous nerves. J Invest Derm, 1977; 69: 130. 